\title{
Diagnostics of Solar Cells
}

\author{
Valeriy Martynyuk ${ }^{1, *}$, Juliy Boiko ${ }^{1}$, Marcin Łukasiewicz ${ }^{2}, E w a$ Kuliśs $^{2}$ and Janusz Musiał ${ }^{2}$ \\ ${ }^{1}$ Programming and Computer, Telecommunication Systems Faculty in Khmelnytsky National \\ University, Department of Telecommunication and Computer Integrated Technology, 29016, 11, \\ Instytuts'ka str., Khmelnitsky, Ukraine \\ ${ }^{2}$ Faculty of Mechanical Engineering UTP in Bydgoszcz, Department of Vehicle Engineering, Al. \\ Prof. S.Kaliskiego 7, 85-796 Bydgoszcz, Poland
}

\begin{abstract}
The paper represents the mathematical model for diagnostics of solar cell. The research objectives are the problem of determining a solar cell technical condition during its operation. The solar cell diagnostics is based on the mathematical model of solar cells. The single-diode solar cell model is characterized by a slight deviation of the theoretically calculated characteristics from the characteristics of the real solar cell, one of the reasons being the complexity of the accurate measurement of the series resistance. The single-diode solar cell model uses the current and voltage ratio in the form of an implicit function and it cannot be solved directly. For its solution it is necessary to use numerical methods. This is main disadvantage of the single-diode solar cell model. The methodological approach to increasing the reliability of the solar cell diagnostic has been proposed, in terms of multi-parameter the solar cell diagnostic by applying the solar cell impedance model.
\end{abstract}

\section{Introduction}

The problem of finding alternative energy sources has become particularly urgent over the last few decades. The reasons for this are the lack of extraction of fossil fuels, primarily oil and gas, as well as environmental pollution caused by their use. On the other hand, the energy of solar radiation is a free, almost everywhere available resource, which allows generating environmentally friendly electricity without the least damage to the environment. In this context, solar energy is one of the most promising sectors of rapidly developing alternative energy.

Today, semiconductor devices with p-n-junctions, which are called solar or photovoltaic (PV) cells, are widely used in the field of solar energy. In such devices, due to the internal photoelectric effect under the influence of solar radiation, there is a redistribution of charges that results in the appearance of electromotive force (EMF), which makes it possible to directly convert the energy of sun light into electrical energy [1,2].

The quality and reliability of solar cells are largely determined by diagnostics support at all stages of their life cycle. At the production stages, where it is crucial to ensure zerodefect manufacturing technology and labour for producing solar cells, the volume of control and diagnostic operations reaches $50 \%$ of the total labour intensity of their production. A characteristic problem of diagnosing solar cell manufacturing defects under the conditions of their mass production is that the later the defects are detected, the more costs are necessary to localize the place of their emergence [3,4].

Hence, diagnostic support will be effective only when the high reliability values are ensured at minimum cost. At the operation stage, the purpose of diagnosing a solar cell is to evaluate its technical condition, to determine the reasons of its operation failures, to predict changes in a solar cell technical condition and determine the reasons for such changes or

\footnotetext{
* Corresponding author: martynyuk.valeriy@gmail.com
} 
time intervals after which processes causing undesirable changes in a solar cell technical condition might begin.

At present, the renewable energy sources are the most important issues of modern energetics that is described in [5]. Thus, the issues of developing methods and means of solar cell diagnostics have become urgent.

Solar cell diagnostics is an information procedure the purpose of which is to reflect its technical condition in the form of a conclusion about the nature and significance of this condition. Such a conclusion regarding the diagnostic results is called a diagnosis [3].

Technical condition of a solar cell is a condition that is characterized at a certain time, under certain environmental conditions by the values of solar cell parameters set by its data sheet. According to the existing practice, it has been confirmed to define such mutually exclusive incompatible pairs of technical conditions for technical products: serviceable unserviceable, operable - faulty.

For the serviceable - unserviceable conditions the basis of their comparison is compliance of all solar cell parameters with the requirements of normative data sheet. And if at least one of these parameters, regardless of its impact on the solar cell ability to perform its functions, will not fulfill these requirements, the technical condition is considered unserviceable. And when all the parameters meet all the requirements of proper documentation, the technical condition is serviceable.

A solar cell is in an operable technical condition if its relevant diagnostic parameters are within the limits where working functions can be performed. When the diagnostic parameters go beyond the specified limits, then the solar cell can no longer fully perform its functions, and it has to be considered faulty.

\section{Solar cell mathematical model}

The single-diode solar cell model received widespread application [1, 2]. It provides an opportunity to perform calculations of the illuminated current-voltage characteristic of a separate panel as well as an array (string) of solar cells connected in series at different values of solar irradiance and the surface temperature of the panel.

An equivalent circuit that corresponds to a single-diode solar cell model is shown in fig. 1. The circuit consists of a current source, one diode, a series and a parallel resistance. The typical current-voltage and power-voltage characteristics of solar cell are shown in fig. 2. The single-diode model in fig. 1 is characterized by a slight deviation of the theoretically calculated characteristics from the characteristics of the real solar cell, one of the reasons being the complexity of the accurate measurement of the series resistance in a solar cell model [2].

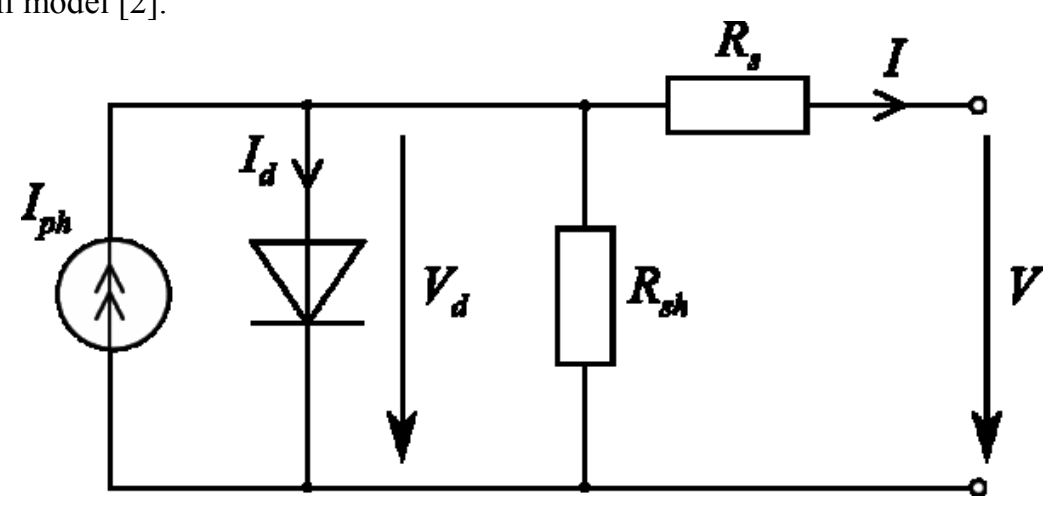

Fig. 1. Solar cell single-diode equivalent circuit. 




Fig. 2. The typical I-V and P-V curves of PV cell.

There are more complicated models that can provide more accurate description of the solar cell characteristics [4]. For example, it is possible to use the model with two diodes [5], one of which simulates the effect of recombination of charges. According to [5], the two-diode model quite accurately describes crystalline silicon solar cells, but for thin-film solar cells the accuracy of approximation may have some limitations. On the other hand, the use of the two-diode model leads to an increase in the number of unknown parameters, which complicates the calculation of the model. So, in this paper the single-diode model of the solar cell is preferred.

The question of choosing an adequate mathematical model for calculating the I-V curves of the solar cell is considered in the following papers [2]. In the paper [5] the problems of simulating I-V curves of solar cells are considered. The authors describe in detail different models of solar cell, analyze the conditions of their application and the accuracy of I-V curves simulation, depending on the solar irradiance and temperature. The single-diode and two-diode solar cell models are compared in works [4-7].

The input data for calculating the solar cell parameters are the value of its I-V curve at three characteristic points corresponding to the short circuit, open circuit and maximum power point (MPP) at the solar irradiance $G=1 \mathrm{~kW} / \mathrm{m}^{2}$ and solar cell surface temperature $T=25{ }^{\circ} \mathrm{C}$. These values of irradiance and temperature correspond to the so-called standard test conditions (STC).

The values of short-circuit current $I_{s c 0}$, open-circuit voltage $U_{s c 0}$, as well as current and voltage at the maximum power point $P_{m p 0}$ under STC, are usually provided by solar panel manufacturers together with the corresponding temperature coefficients. Series resistance $R_{s}$, parallel resistance $R_{s h}$, photocurrent $I_{p h}$, as well as saturation current $I_{s}$ and ideality factor for a diode $m$ remain unknown $[8,9]$.

There are quite a lot of methods for calculating the unknown solar cell parameters, which differ in the approaches to the solution of the I-V equation given in an implicit form. For example, paper [4] describes a method based on the decomposition of the I-V equation for a solar cell into the Taylor series. In paper [6], the authors present a methodology for calculating solar module parameters based on the datasheet information. Paper [6] is devoted to the development of a solar cell model in the Matlab/Simulink software environment. Paper [7] presents a method for calculating series and parallel resistances of single-diode solar cell model on the basis of I-V curve analysis.

In this paper, a detailed description of the procedure for calculating the parameters of a single-diode solar panel model is provided. The algorithm for calculating series and parallel resistances of the solar cell equivalent circuit is presented. On the basis of the obtained ratios, a simulation model of solar cell in Matlab/Simulink software environment was developed.

Basing on Kirchhoff's laws, we can write the following expression for the circuit in fig. 1: 


$$
I=I_{p h}-I_{d}-\frac{V+I R_{s}}{R_{s h}}
$$

where: $I$ and $V$ are solar cell output current and voltage; $I_{p h}$ is photocurrent; $I_{d}$ is current flowing through the diode; $R_{s}$ is series resistance; $R_{s h}$ is parallel (shunt) resistance.

The expression for the current $I_{d}$ can be obtained on the basis of the ideal p-n-junction equation [2-5]. For the array consisting of $N_{s}$ PV cells connected in series, current $I_{d}$ is defined by the following ratio:

$$
I_{d}=I_{s}\left[\exp \left(\frac{V_{d}}{m V_{T}}\right)-1\right]
$$

where: $I_{s}$ is reverse saturation current; $I_{d}=V+I R_{s}$ is voltage at the diode; $V_{T}$ is thermal voltage; $m$ is diode ideality factor.

The value of the thermal voltage $V_{T}$ is defined by the following expression:

$$
V_{T}=\frac{N_{s} k T}{q}
$$

where: $N_{s}$ is the number of solar cells connected in series; $T$ is absolute p-n-junction temperature; $k$ is Boltzmann constant; $q$ - elementary charge equal to the module of electron charge.

Thus, taking to account (1) - (3), the analytical expression for the I-V curve of an array of $N_{s}$ solar cells connected in series is the following:

$$
I=I_{p h}-I_{s}\left[\exp \left(\frac{V+I R_{s}}{m V_{T}}\right)-1\right]-\frac{V+I R_{s}}{R_{s h}}
$$

The model, represented by expression (4), allows us to develop theoretical I-V curves of solar panels with an accuracy that is sufficient for engineering calculations and computer simulation [2].

It includes 5 unknown parameters that have to be found in different ways: $I_{p h}, I_{s}, m$, $R_{s}$ and $R_{s h}$. There are many methods for defining these parameters, most of which are based on an assumption that $R_{s}$ takes low values, but $R_{s h}$, on the contrary, is quite large.

Thus, assigning $R_{s}=0$ and $R_{s h}=\infty$ in expression (4), we can get a simplified expression for I-V characteristic:

$$
I=I_{p h}-I_{s}\left[\exp \left(\frac{V}{m V_{T}}\right)-1\right]
$$

Expression (5) corresponds to the model of the ideal solar cell. It allows representing I$\mathrm{V}$ characteristic in the form of explicit function $I=I(V)$, but with less precision than in expression (4) $[4,5]$.

The coefficient $m$ is different for each material a solar cell is made of. For example, for the solar cells made of polycrystalline silicon $m=1.3$ [9].

Let's consider I-V curve of solar cell at the point $\left(0, I_{s c 0}\right)$, which corresponds to short circuit (fig. 2).

Substituting these values into equation (5), we obtain the approximate value of the photocurrent under the STC:

$$
I_{p h 0} \approx I_{s c 0}
$$


where: $I_{s c 0}$ is short-circuit current under the test conditions.

Let's represent the exact photocurrent value $I_{p h 0}$ by the following expression:

$$
I_{p h 0}=\alpha I_{s c 0}
$$

where: $\alpha$ is the coefficient of proportionality.

The actual photocurrent value $I_{p h}$ depends on the temperature of the panel and solar irradiance, and, taking into account (6), is defined by the following ratio $[8,9,10]$ :

$$
I_{p h}=\frac{G}{G_{o}}\left[1-K_{I}\left(T-T_{o}\right)\right] I_{p h 0}
$$

where: $G_{0}=1 \mathrm{~kW} / \mathrm{m}^{2}$ is the solar irradiance under the STC; $T_{0}=25^{\circ} \mathrm{C}$ is the solar cell temperature under the STC; $K_{\mathrm{I}}$ is the temperature coefficient of short circuit.

Let's consider I-V curve at the point $\left(V_{o c 0}, 0\right)$, which corresponds to solar cell open circuit (fig. 2). Substituting these values into equation (5) and taking into account (6) we obtain the expression for the value of the diode saturation current under the STC:

$$
I_{s 0}=\frac{I_{s c 0}}{\exp \left(\frac{V_{o c 0}}{m V_{T o}}\right)-1}
$$

where: $V_{o c 0}$ and $V_{T 0}$ are open-circuit voltage and thermal voltage under the STC correspondingly.

The dependence of the current $I_{s}$ on the temperature can be expressed with the help of temperature coefficients of short circuit and open circuit [8] according to the following ratio:

$$
I_{s}=\frac{I_{s c 0}\left(1+K_{I} \Delta T\right)}{\exp \left(\frac{V_{o c 0}\left(1+K_{V} \Delta T\right)}{m V_{T}}\right)-1}
$$

where: $\Delta T=T-T_{0} ; K_{V}$ is open-circuit temperature coefficient.

For the mathematical model (4), resistances $R_{s}$ and $R_{s h}$ remain unknown. They can be defined on the basis of the equality of the experimental and theoretical maximum power values.

It is known that the solar cell output power is a product of output voltage $V$ and current $I$ :

$$
P=V \cdot I
$$

where $I$ and $V$ are PV panel output current and voltage correspondingly.

Let's substitute $I=I_{m p}$ and $V=V_{m p}$ into the equation (4) and, taking into account expression (11), write the expression which represents the ratio between $R_{s h}$ and $R_{s}$ :

$$
R_{s h}=\frac{V_{m p}+I_{m p} R_{s}}{I_{p h}-I_{s}\left[\exp \left(\frac{V_{m p}+I_{m p} R_{s}}{m V_{T}}\right)-1\right]-I_{m p}}
$$

From equation (12), for some $R_{s}$ we can find the corresponding value of $R_{s h}$, at which the theoretical I-V curve will pass through the experimental MPP. Gradually increasing the value of $R_{s}$, starting from $R_{s}=0$, it's possible to find such a pair of $\left[R_{s}, R_{s h}\right]$ at which the theoretical MPP will be equal (with the known error) to the experimental one. To achieve this, for each pair of $\left[R_{s}, R_{s h}\right]$ from equations (4) and (12) we should find the maximum power value $P$ which corresponds to the theoretical I-V curve. Thus, on each iteration of the 
algorithm the values of $R_{s}$ and $R_{s h}$ are approaching the optimal ones, that provide the best possible accuracy of the model.

The initial value of resistance $R_{s h}$ can be defined according to the following expression [8]:

$$
R_{s h 0}=\frac{V_{m p}}{I_{s c 0}-I_{m p}}-\frac{V_{o c 0}-V_{m p}}{I_{m p}}
$$

where: $I_{m p}$ and $V_{m p}$ are current and voltage corresponding to the experimental MPP.

As shown in expression (6), $I_{p h 0} \neq I_{s c 0}$. The photocurrent value $I_{p h 0}$ under the STC can be expressed with higher accuracy if to put $\alpha=1+\frac{R_{s}}{R_{s h}}$ into expression (7). So, the final expression for the photocurrent $I_{p h 0}$ looks like this:

$$
I_{p h 0}=\left(1+\frac{R_{s}}{R_{s h}}\right) I_{s c 0}
$$

Since equation (4) sets the current and voltage ratio in the form of an implicit function $f=f(I, V)$, it cannot be solved directly. For its solution it is necessary to use numerical methods [7].

\section{Solar cell diagnostics system}

In terms of electrical engineering, a solar cell is an active bi-polarity with frequency dispersion of its electrical parameters. According to the theory of mathematical modeling, any mathematical model can be represented graphically and analytically.

Proceeding from this, under the impedance mathematical model of the solar cell in graphical form, we mean its equivalent circuit on alternating current. In analytical form, the impedance mathematical model of the solar panel is the analytical expression of its impedance, which combines the parameters of the equivalent circuit elements with the electrical parameters of the solar panel.

To determine the solar cell parameters we conducted the solar cell impedance measurements by means of the solar cell diagnostic system which is shown in fig. 3 .

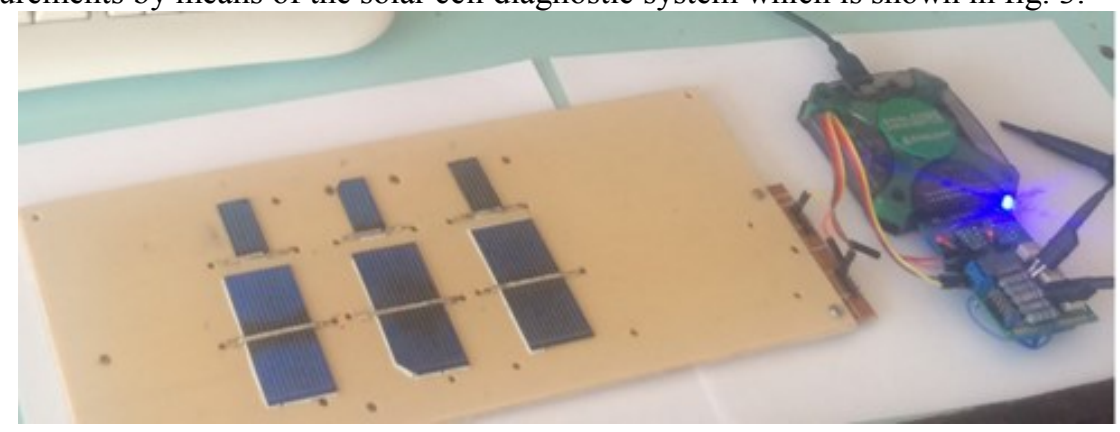

Fig. 3. Solar cell diagnostic system.

Structurally, the solar cell diagnostic system consists of an Analog Discovery 2 device produced by the US Digilent which is shown in fig. 4, a. To measure the impedance of solar cells, the specialized Wave Forms software by the US Digilent was used as shown in fig. 4 , b. 


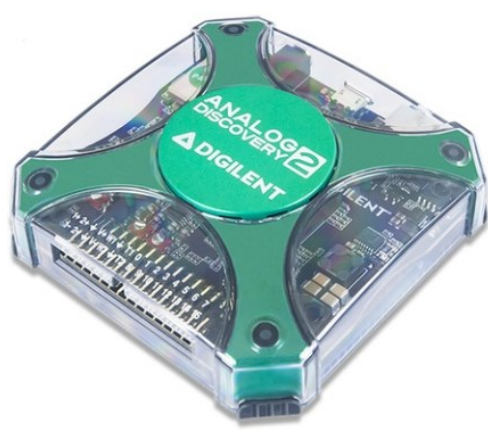

a)

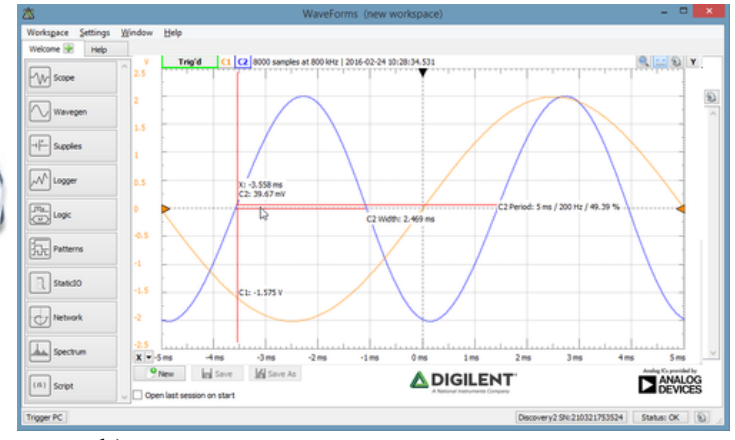

b)

Fig. 4. The picture of Analog Discovery 2 and Wave Forms software

Analog Discovery 2 is connected to an adapter assembled on a model board and laboratory bench as shown in fig. 5 .

Digital outputs of the Analog Discovery 2 are used to connect one of the reference resistances needed to measure current through a solar cell. The solar cell data acquisition system allows estimating impedance in the solar cell from $1 \Omega$ to $1 \mathrm{M} \Omega$ in the frequency range from $1 \mathrm{~Hz}$ to $1 \mathrm{MHz}$ with a regular frequency distribution within the range.

The experimental measurements of impedance in the solar cell performed by the authors show that its experimental Nyquist plot has the form of two semicircles, as shown in fig. 6 . The large semicircle corresponds to the barrier capacitance $C_{\mathrm{b}}$ of the solar cell, the effect of which is observed at high frequencies (from $100 \mathrm{~Hz}$ to $1 \mathrm{MHz}$ ).

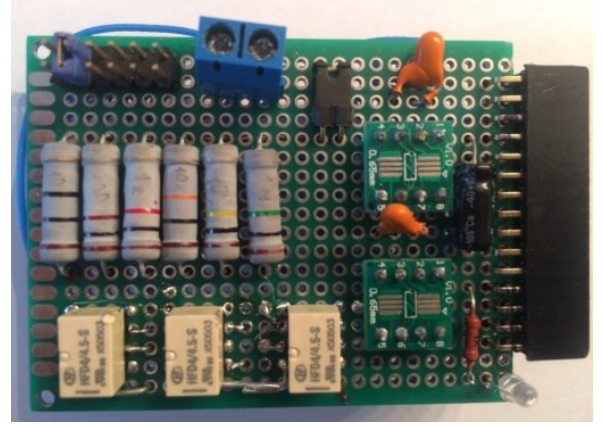

Fig. 5. The picture of adapter.

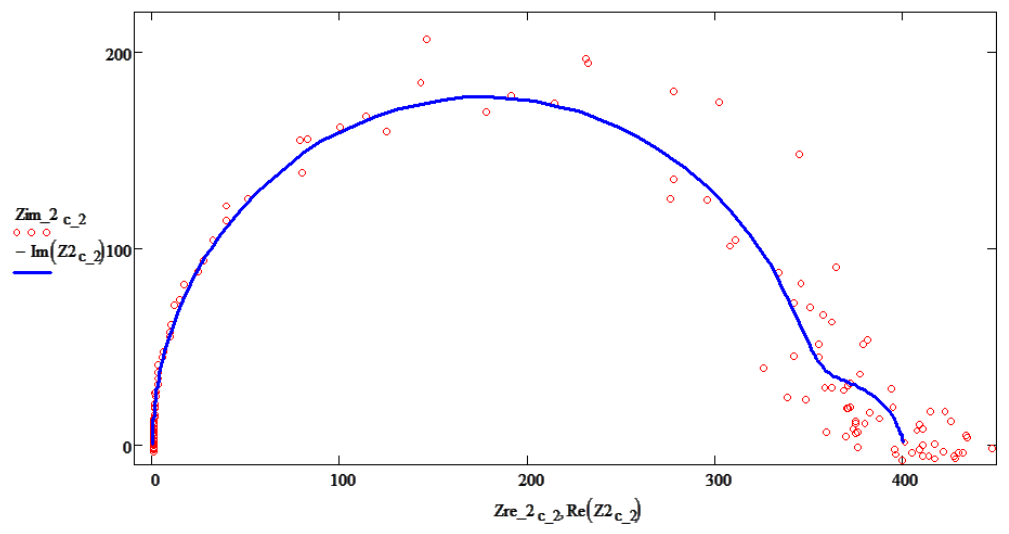

Fig. 6. Experimental and theoretical Nyquist plots of the solar cell. 
The small semicircle corresponds to diffusion capacitance $C_{\mathrm{d}}$ and frequency-dependent conductivity the influence of which is observed at low frequencies (from $1 \mathrm{~Hz}$ to $100 \mathrm{~Hz}$ ). By analysing the experimental Nyquist plot as shown in fig. 6 we can conclude that the small semicircle is substantially deformed by the loss of electric energy and is under the influence of noise obstruction. Such a character of the Nyquist plot corresponds to the fractional capacitor with significant losses of electric energy [7, 8].

Thus, the authors suggest an impedance mathematical model of the solar cell, related to the expression of impedance (15).

$$
Z(s)=R_{\mathrm{s}}+\frac{R_{\mathrm{b}}}{1+s C_{\mathrm{b}} R_{\mathrm{b}}}+\frac{R_{\mathrm{d}}}{1+s^{\alpha} C_{\mathrm{d}} R_{\mathrm{d}}}
$$

The adequacy of the suggested impedance mathematical model of the solar cell is confirmed by the convergence of the experimental and theoretical Nyquist plots, which are shown in fig. 6.

By analysing the expression (15) of impedance the authors propose the solar cell equivalent circuit as shown in fig. 7. The solar cell equivalent circuit consisting of series resistance $R_{\mathrm{s}}$, barrier capacitance $C_{\mathrm{b}}$, barrier resistance $R_{\mathrm{b}}$, diffusion resistance $R_{\mathrm{d}}$ and diffusion capacitance $C_{\mathrm{d}}$ of the fractional order.



Fig. 8. The impedance solar cell equivalent circuit.

As a result of the approximation of the experimental Nyquist plot the following values of the parameters of the improved solar cell equivalent circuit have been obtained: $R_{\mathrm{s}}=0,1 \Omega$, $C_{\mathrm{b}}=200 \mathrm{nF}, R_{\mathrm{b}}=350 \Omega, C_{\mathrm{d}}=100 \mu \mathrm{F}, R_{\mathrm{d}}=50 \Omega, \alpha=0,9$.

Next, two hypotheses are put forward.

1. The basic hypothesis $\mathrm{H}_{0}$ (the solar cell is working):

$$
\begin{aligned}
R s & \in\left[R s_{\min }, R s_{\max }\right] ; \\
R b & \in\left[R b_{\min }, R b_{\max }\right] ; \\
R d & \in\left[R d_{\min }, R d_{\max }\right] ; \\
C d & \in\left[C d_{\min }, C d_{\max }\right] ; \\
\alpha & \in\left[\alpha_{\min }, \alpha_{\max }\right] .
\end{aligned}
$$

where: $\mathrm{Rs}_{\min }, \mathrm{Rs}_{\max }$; $\mathrm{Rb}_{\text {min }}, \mathrm{Rb}_{\max }$; $\mathrm{Rd}_{\min }, \mathrm{Rd}_{\max } ; \mathrm{Cd}_{\min }, \mathrm{Cd}_{\max }$ and $\alpha_{\min }, \alpha_{\max }$ are limits of permissible interval for $\mathrm{Rs}, \mathrm{Rb}$; $\mathrm{Rd}, \mathrm{Cd}$ and $\alpha$ respectively.

2. Alternative hypothesis $\mathrm{H}_{1}$ (the solar cell is defective):

$$
\begin{aligned}
R s & \notin\left[R s_{\min }, R s_{\max }\right] ; \\
R b & \notin\left[R b_{\text {min }}, R b_{\text {max }}\right] ; \\
R d & \notin\left[R d_{\text {min }}, R d_{\max }\right] ; \\
C d & \notin\left[C d_{\min }, C d_{\max }\right] ; \\
\alpha & \notin\left[\alpha_{\min }, \alpha_{\max }\right] .
\end{aligned}
$$

The diagnostic reliability of the solar cell is defined as the product of the methodological and the instrumental diagnostic reliabilities of the solar cell. The 
methodological diagnostic reliability of the solar cell is defined by the ratio between the number of exponents characterizing the technical condition of the object of diagnostic and included to the mathematical model of diagnostics and the total number of exponents characterizing the technical condition of the solar cell.

The instrumental diagnostic reliability of the solar cells is defined by the probabilities of errors of the first and second kind, $\alpha$ and $\beta$ respectively. Increase in the diagnostic reliability of the solar cell is possible when using the solar cell characteristics that contain information on the nominal values of technical condition of the solar cell in the range of changes in nominal voltages in the operation current range. Such characteristics include nonlinear resistance $Z(s, u)$ and nonlinear transient resistance $z(t, u)$ of the solar cells, related to each other by inverse and direct Laplace transforms [9-11]:

$$
\begin{aligned}
& Z(s, u)=L^{-1}[z(t, u)] ; \\
& z(t, u)=L[Z(s, u)],
\end{aligned}
$$

where: $s=j \omega$ is complex frequency.

\section{Conclusions}

Solar cells are the basis of solar power plants. Each year, the production of solar cells is increasing, so their diagnostics is very important to the electric energy production. The solar cell diagnostics is based on the mathematical model of solar cells. The single-diode solar cell model is characterized by a slight deviation of the theoretically calculated characteristics from the characteristics of the real solar cell, one of the reasons being the complexity of the accurate measurement of the series resistance. The single-diode solar cell model uses the current and voltage ratio in the form of an implicit function and it cannot be solved directly. For its solution it is necessary to use numerical methods. This is main disadvantage of the ingle-diode solar cell model.

To solve this disadvantage the authors propose to use the impedance solar cell model. The impedance solar cell model is based on the series resistance $R_{\mathrm{s}}$, barrier capacitance $C_{\mathrm{b}}$, barrier resistance $R_{\mathrm{b}}$, diffusion resistance $R_{\mathrm{d}}$ and diffusion capacitance $C_{\mathrm{d}}$ of the fractional order. These parameters were obtained from the solar cell experimental impedance data. The solar cell impedance was measured by means of the solar cell diagnostic system which is developed by authors.

\section{References}

1. T. Ahmad, S. Sobhan, S. Performance Study of Photovoltaic Solar Cell. International Jour. of Research in Engineering and Technology, V. 4, pp. 12-18, (2015)

2. C. Carrero, J. Amador, S. Arnaltes. A Single Procedure for Helping PV Designers to Select Silicon PV Modules and Evaluate the Loss Resistances. Renewable Energy, V. 32, pp. 2579-2589, (2007)

3. V. Martynyuk, O. Eromenko, J. Boiko, T. Kałaczyński. Diagnostics of supercapacitors. MATEC Web Conf., 182, 01009, (2018)

4. P. Arjyadhara1, Ali S.M, J. Chitralekha. Analysis of Solar PV cell Performance with Changing Irradiance and Temperature. Inter. Jour. of Engineering and Computer Science, V. 2, I. 1, pp. 214-220, (2013)

5. M. G. Villalva, J. R. Gazoli, E. Ruppert F. Modeling and circuit-based simulation of photovoltaic arrays. Brazilian Jour. of Power Electronics, V. 14, no. 1, pp. 35-45, (2009)

6. H. Bellia, R. Youcef, M. Fatima. A detailed modeling of photovoltaic module using MATLAB. NRIAG Jour. of Astronomy and Geophysics, pp.53-61, (2014) 
7. M. Ortigueira, J. Trujillo, V. Martynyuk, J. Coito. A generalized power series and its application in the inversion of transfer functions. Jour. of Signal Processing, V. 107, pp. 238-245, (2015)

8. V. De Santis, V. Martynyuk, A. Lampasi, M. Fedula, M. Ortigueira. Fractional-order circuit models of the human body impedance for compliance tests against contact currents. AEU - Inter. Jour. of Electr. and Communcations, V. 78, pp. 238-244, (2017)

9. V. Martynyuk, D. Makaryshkin, J. Boyko, "Frequency domain analysis for electrochemical supercapacitors", in 2007 Proc. 15th IMEKO Symposium on Novelties in Electrical Measurements and Instrument. in Parallel with the 12th Workshop on ADC Modelling and Testing, Iasi, pp. 5, (2007)

10. V. Martynyuk, D. Makaryshkin, J. Boyko, "Electrochemical supercapacitor time domain analysis by means of multi-channel measurement system", in 2007 Proc. 15th IMEKO Symposium on Novelties in Electrical Measurements and Instrument. in Parallel with the 12th Workshop on ADC Modelling and Testing, Iasi, 2007, Vol. I, pp. 207-211, (2007)

11. T. Kałaczyński, M. Łukasiewicz, J. Musiał, R. Polasik, M. Szczutkowski, N. Dluhunovych, J. Wilczarska, T. Kasprowicz, Analysis of the diagnostic potential research thermovision in the technical state of combustion engine injectors assessment, Engineering Mechanics, 1805-8248, 357-360, (2018) 